\title{
Well-separated spherical designs
}

\author{
Andriy Bondarenko*, Danylo Radchenko, and Maryna Viazovska
}

\begin{abstract}
For each $N \geq C_{d} t^{d}$ we prove the existence of a well-separated spherical $t$-design in the sphere $S^{d}$ consisting of $N$ points, where $C_{d}$ is a constant depending only on $d$.
\end{abstract}

Keywords: spherical designs, well-separated configurations, topological degree, Marcinkiewicz-Zygmund inequality, area-regular partitions.

AMS subject classification. 52C35, 41A55, 41A05, 41A63

\section{Introduction}

In this paper we will discuss the interrelation between several classical optimization problems on spheres $S^{d}$ such as minimal equal-weight quadratures (spherical designs), best packing problems, and minimal energy problems. For $d=1$, a regular polygon is an optimal configuration for all of these problems. However, for $d \geq 2$ exact solutions are known in very few cases. Even asymptotically optimal configurations are sometimes very hard to obtain (see for example Smale's 7th Problem [18]).

We will prove the existence of certain configurations in $S^{d}$ which are spherical $t$-designs with asymptotically minimal number of points and that

*This work was carried out during the tenure of an ERCIM "Alain Bensoussan" Fellowship Programme. The research leading to these results has received funding from the European Union Seventh Framework Programme (FP7/2007-2013) under grant agreement $n^{\circ} 246016$. 
simultaneously have asymptotically the best separation property. These configurations also provide approximate solutions for several other optimization problems.

Let $S^{d}=\left\{x \in \mathbb{R}^{d+1}:|x|=1\right\}$ be the unit sphere in $\mathbb{R}^{d+1}$ equipped with the Lebesgue measure $\mu_{d}$ normalized by $\mu_{d}\left(S^{d}\right)=1$. A set of points $x_{1}, \ldots, x_{N} \in S^{d}$ is called a spherical t-design if

$$
\int_{S^{d}} P(x) d \mu_{d}(x)=\frac{1}{N} \sum_{i=1}^{N} P\left(x_{i}\right)
$$

for all polynomials in $d+1$ variables, of total degree at most $t$. The concept of a spherical design was introduced by Delsarte, Goethals, and Seidel [9]. For each $d, t \in \mathbb{N}$ denote by $N(d, t)$ the minimal number of points in a spherical $t$-design in $S^{d}$. The following lower bound

$$
N(d, t) \geq \begin{cases}\left(\begin{array}{c}
d+k \\
d
\end{array}\right)+\left(\begin{array}{c}
d+k-1 \\
d
\end{array}\right) & \text { if } t=2 k, \\
2\left(\begin{array}{c}
d+k \\
d
\end{array}\right) & \text { if } t=2 k+1,\end{cases}
$$

is proved in [9] (see also the classical monograph [8]). On the other hand, it follows from the general result by Seymour and Zaslavsky [17] that spherical designs exist for all positive integers $d$ and $t$. The method of proof used in [17] was not constructive and authors did't indicate an upper bound for $N(d, t)$ in terms of $d$ and $t$. First feasible upper bounds were given by Wagner [19] $\left(N(d, t) \leq C_{d} t^{C d^{4}}\right)$ and Bajnok [3] $\left(N(d, t) \leq C_{d} t^{C d^{3}}\right)$. Korevaar and Meyers [12] have improved these inequalities by showing that $N(d, t) \leq C_{d} t^{\left(d^{2}+d\right) / 2}$. They have also conjectured that $N(d, t) \leq C_{d} t^{d}$. Note that (1) implies $N(d, t) \geq c_{d} t^{d}$. Here and in what follows we use the notations $C_{d}, L_{d}$, etc. $\left(c_{d}, \lambda_{d}\right.$, etc.) for sufficiently large (small) constants depending only on $d$.

Korevaar and Meyers were motivated by the following problem coming from potential theory: How to choose $N$ equally charged points $x_{1}, \ldots, x_{N}$ 
in $S^{2}$ to minimize the value

$$
U_{r}\left(x_{1}, \ldots, x_{N}\right):=\sup _{|x|=r}\left|\frac{1}{N} \sum_{i=1}^{N} \frac{1}{\left|x-x_{i}\right|}-1\right|, \quad r \in(0,1) ?
$$

The classical Faraday cage phenomenon states that any stable charge distribution on the compact closed surface cancels the electric field inside the surface. According to this model the minimal value of $U_{r}$ should rapidly decay to 0 , when $N$ grows.

It was shown in [12] that if the set of points $x_{1}, \ldots, x_{N}$ is a spherical $t$-design for some $t>c N^{1 / 2}$ then

$$
U_{r}\left(x_{1}, \ldots, x_{N}\right) \leq r^{\alpha N^{1 / 2}}
$$

The estimate is optimal up to the constant in the power.

Recently we have suggested a nonconstructive approach to obtain an optimal asymptotic bound for $N(d, t)$ based on the application of the topological degree theory; see [4, 5]. We have proved the following

Theorem A. For each $N \geq C_{d} t^{d}$ there exists a spherical $t$-design in $S^{d}$ consisting of $N$ points.

This implies the Korevaar-Meyers conjecture.

Now we will give the definition of a well-separated sequence of configurations. A sequence of $N$-point configurations $X_{N}=\left\{x_{1 N}, \ldots, x_{N N}\right\}$ in $S^{d}$ is called well-separated if

$$
\min _{1 \leq i<j \leq N}\left|x_{i N}-x_{j N}\right| \geq \lambda_{d} N^{-1 / d}
$$

for some constant $\lambda_{d}$ and all $N \geq 2$. The inequality (2) is optimal up to the constant $\lambda_{d}$. That is, there exists a constant $L_{d}$ such that for any $N$-point configuration $\left\{x_{1}, \ldots, x_{N}\right\}$

$$
\min _{1 \leq i<j \leq N}\left|x_{i}-x_{j}\right|<L_{d} N^{-1 / d}
$$

Many authors have predicted the existence of well-separated spherical $t$ designs in $S^{d}$ of asymptotically minimal cardinality $O\left(t^{d}\right)$ as $t \rightarrow \infty$ (see, 
e.g. 2] and [11]). Moreover, in [11] it was shown that if such spherical designs exist then they have asymptotically minimal Riesz $s$-energy. In this paper we prove the existence of above mentioned spherical designs. Our main result is:

Theorem 1. For each $d \geq 2$ there exist positive constants $C_{d}$ and $\lambda_{d}$ depending only on d such that for each $t \in \mathbb{N}$ and each $N>C_{d} t^{d}$ there exists a spherical t-design in $S^{d}$ consisting of $N$ points $\left\{x_{i}\right\}_{i=1}^{N}$ with $\left|x_{i}-x_{j}\right| \geq \lambda_{d} N^{-1 / d}$ for $i \neq j$.

Theorem 1 is a natural generalization of Theorem A. The paper is organized as follows. In Section 2 we will reduce Theorem 1 to the construction of a certain $N$-tuple of maps $\mathbf{x}_{1}, \ldots, \mathbf{x}_{N}: \mathcal{P}_{t} \rightarrow S^{d}$. Then in Section 3 we will prove several auxiliary results concerning area regular partitions of sphere $S^{d}$ and spherical Marcinkiewicz-Zygmund type inequalities. Finally in Section 4 we will construct the maps $\mathbf{x}_{1}, \ldots, \mathbf{x}_{N}$, proving Theorem 1 .

\section{Application of topological degree theory}

We will use the approach similar to that of [4]. Let $\mathcal{P}_{t}$ be the Hilbert space of polynomials $P$ of degree at most $t$ on $S^{d}$ such that

$$
\int_{S^{d}} P(x) d \mu_{d}(x)=0
$$

equipped with the usual inner product

$$
(P, Q)=\int_{S^{d}} P(x) Q(x) d \mu_{d}(x) .
$$

By Riesz representation theorem, for each point $x \in S^{d}$ there exists a unique polynomial $G_{x} \in \mathcal{P}_{t}$ such that

$$
\left(G_{x}, Q\right)=Q(x) \text { for all } Q \in \mathcal{P}_{t}
$$

Then a set of points $x_{1}, \ldots, x_{N} \in S^{d}$ forms a spherical $t$-design if and only if

$$
G_{x_{1}}+\cdots+G_{x_{N}}=0
$$


The gradient of a differentiable function $f: \mathbb{R}^{d+1} \rightarrow \mathbb{R}$ is denoted by

$$
\frac{\partial f}{\partial x}:=\left(\frac{\partial f}{\partial \xi_{1}}, \ldots, \frac{\partial f}{\partial \xi_{d+1}}\right), \quad x=\left(\xi_{1}, \ldots, \xi_{d+1}\right) .
$$

For a polynomial $Q \in \mathcal{P}_{t}$ we define the spherical gradient

$$
\nabla Q(x):=\frac{\partial}{\partial x}\left(Q\left(\frac{x}{|x|}\right)\right) .
$$

Now we will use the following result from topological degree theory [15, Ths. 1.2.6 and 1.2.9].

Theorem B. Let $f: \mathbb{R}^{n} \rightarrow \mathbb{R}^{n}$ be a continuous map and $\Omega$ an open bounded subset, with boundary $\partial \Omega$, such that $0 \in \Omega \subset \mathbb{R}^{n}$. If $(x, f(x))>0$ for all $x \in \partial \Omega$, then there exists $x \in \Omega$ satisfying $f(x)=0$.

We will apply Theorem $B$ to the following open subset of a vector space $\mathcal{P}_{t}$

$$
\Omega=\left\{P \in \mathcal{P}_{t}\left|\int_{S^{d}}\right| \nabla P(x) \mid d \mu_{d}(x)<1\right\} .
$$

Observe that if continuous maps $\mathbf{x}_{i}: \mathcal{P}_{t} \rightarrow S^{d}, i=1, \ldots, N$, satisfy for all $P \in \partial \Omega$

$$
\sum_{i=1}^{N} P\left(\mathbf{x}_{i}(P)\right)>0
$$

then there exists a spherical $t$-design on $S^{d}$ consisting of $N$ points. To this end let us consider a map $f: \mathcal{P}_{t} \rightarrow \mathcal{P}_{t}$ defined by

$$
f(P)=G_{\mathbf{x}_{1}(P)}+\cdots+G_{\mathbf{x}_{N}(P)} .
$$

Clearly

$$
(P, f(P))=\sum_{i=1}^{N} P\left(\mathbf{x}_{i}(P)\right)
$$

for each $P \in \mathcal{P}_{t}$. Thus, applying Theorem B for the map $f$, vector space $\mathcal{P}_{t}$, and the subset $\Omega$ defined in (41) immediately gives us the existence of a polynomial $\widetilde{P} \in \Omega$ such that $f(\widetilde{P})=0$. Hence, by (3) , the images of this polynomial $\mathbf{x}_{1}(\widetilde{P}), \ldots, \mathbf{x}_{N}(\widetilde{P})$ form a spherical $t$-design in $S^{d}$ consisting of $N$ 
points. If additionally there exists a constant $\lambda_{d}$ such that $\left|\mathbf{x}_{i}(P)-\mathbf{x}_{j}(P)\right| \geq$ $\lambda_{d} N^{-1 / d}$ for all $i \neq j$, and $P \in \Omega$, then the above mentioned spherical $t$-design is well separated, proving Theorem 1 .

The maps $\mathbf{x}_{i}, i=1, \ldots, N$, will be constructed in Section 4 below.

\section{Area-regular partitions and convex sets}

For $x, y \in S^{d}$ denote by $\operatorname{dist}(x, y)=\arccos ((x, y))$ the geodesic distance between $x$ and $y$. Also for a set $A \subset S^{d}$ define the geodesic distance between $x$ and $A$ as follows

$$
\operatorname{dist}(x, A)=\inf _{y \in A} \operatorname{dist}(x, y) .
$$

Recall that a spherical cap of radius $r$ with center at $x \in S^{d}$ is the set

$$
A(x, r)=\left\{z \in S^{d} \mid \operatorname{dist}(x, z) \leq r\right\}
$$

Below we will use extensively the notion of an area-regular partition. Here is the definition.

Let $\mathcal{R}=\left\{R_{1}, \ldots, R_{N}\right\}$ be a finite collection of closed sets $R_{i} \subset S^{d}$ such that $\cup_{i=1}^{N} R_{i}=S^{d}$ and $\mu_{d}\left(R_{i} \cap R_{j}\right)=0$ for all $1 \leq i<j \leq N$. The partition $\mathcal{R}$ is called area-regular if $\mu_{d}\left(R_{i}\right)=1 / N, i=1, \ldots, N$. The partition norm for $\mathcal{R}$ is defined by

$$
\|\mathcal{R}\|=\max _{R \in \mathcal{R}} \operatorname{diam} R=\max _{R \in \mathcal{R}} \max _{x, y \in R} \operatorname{dist}(x, y) .
$$

It is easy to prove using isodiametric inequality that each $R_{i}$ has diameter at least $c_{d} N^{-1 / d}$. Therefore, $\|\mathcal{R}\| \geq c_{d} N^{-1 / d}$ for each $\mathcal{R}$. However for some $C_{d}$ and each $N \in \mathbb{N}$ there exists an area-regular partition $\mathcal{R}=\left\{R_{1}, \ldots, R_{N}\right\}$ of $S^{d}$ with diameter at most $C_{d} N^{-1 / d}$. Such area-regular partitions are used for many optimization problems where a well distributed set of $N$ points on a sphere having no concentration points is needed (see e.g. [1], 6], 14], and [13]). In this paper we need area-regular partitions of small diameter with additional constraint of geodesic convexity. A subset $A \subset S^{d}$ is geodesically convex if any two points $x, y \in A$ can be connected by a geodesic arc 
contained in $A$. The partition $\mathcal{R}=\left\{R_{1}, \ldots, R_{N}\right\}$ is said to be convex if each set $R_{i}, i=1, \ldots, N$, is geodesically convex. First we will prove the existence of convex area-regular partitions of "small" diameter.

Proposition 1. For each $N \in \mathbb{N}$ there exists a convex area-regular partition $\mathcal{R}=\left\{R_{1}, \ldots, R_{N}\right\}$ such that $\|\mathcal{R}\| \leq K_{d} N^{-1 / d}$, where $K_{d}$ is a constant depending only on $d$.

The following construction for the sphere $S^{2}$ and $N=6 n^{2}$, where $n \in \mathbb{N}$, is given by Alexander in [1]. Let us first explain his simple and elegant proof.

We begin with a spherical cube, and consider one of its facets. Using $n-1$ great circles from the pencil determined by two opposite edges we can cut the facet into $n$ slices of equal area. Each slice can be cut into $n$ quadrilaterals of equal area using great circles in the pencil in the other pair of opposite edges of the face. The diameters of the quadrilaterals are of the right magnitude.

This construction has an obvious generalization to higher dimensions. Start with the appropriate spherical hypercube, then divide each face into $n$ equal pieces, and so on. In this way we obtain a convex partition of $S^{d}$ into $2(d+1) n^{d}$ parts of diameter at most $C_{d} / n$.

Now we generalize the approach of Alexander to prove Proposition 1 for all $N \in \mathbb{N}$. For each $m \in \mathbb{N}$ and a vector with positive coordinates $a=$ $\left(a_{1}, \ldots, a_{m}\right) \in \mathbb{R}_{+}^{m}$ denote by $P(a)$ the $m$-dimensional rectangle $\left[-a_{1}, a_{1}\right] \times$ $\cdots \times\left[-a_{m}, a_{m}\right]$. Also, a measure $d \eta(x)=\alpha(x) d x$ defined on $P(a)$ is said to be $M$-uniform if

$$
\alpha(x) \leq M \alpha(y) \text { for all } x, y \in P(a)
$$

To prove Proposition 1 we need the following lemma.

Lemma 1. Let $a \in \mathbb{R}_{+}^{m}$ with

$$
\max _{1 \leq i, j \leq m} \frac{a_{i}}{a_{j}} \leq B
$$

and $d \eta$ be a $M$-uniform measure defined on $P(a)$. Then for each $N \in \mathbb{N}$ there exists a partition of $P(a)$ into $N$ m-rectangles $P_{1}, \ldots, P_{N}$ with facets 
parallel to the corresponding facets of $P(a)$ such that

$$
\int_{P_{i}} d \eta(x)=\frac{1}{N} \int_{P(a)} d \eta(x),
$$

and

$$
\operatorname{diam} P_{i} \leq C(m, B, M) a_{1} N^{-1 / m}
$$

for all $i=1, \ldots, N$.

Proof. We will prove the lemma by induction on $m$. For $m=1$, first we choose a point $t_{1} \in\left[-a_{1}, a_{1}\right]$ such that

$$
\int_{-a_{1}}^{t_{1}} d \eta(x)=\frac{1}{N} \int_{-a_{1}}^{a_{1}} d \eta(x) .
$$

Then similarly we choose $t_{2} \in\left(t_{1}, a_{1}\right]$ such that

$$
\int_{t_{1}}^{t_{2}} d \eta(x)=\frac{1}{N} \int_{-a_{1}}^{a_{1}} d \eta(x)
$$

and so on. Finally, we get the partition of $P(a)=\left[-a_{1}, a_{1}\right]$ into $N$ segments $P_{1}=\left[-a_{1}, t_{1}\right], P_{2}=\left[t_{1}, t_{2}\right], \ldots, P_{N}=\left[t_{N-1}, a_{1}\right]$ satisfying (5) by its definition. Moreover, $M$-uniformity of $\eta$ implies (6) with $C(1, B, M)=2 M$. Assume that the lemma is true for $m=l-1$. Let us prove it for $m=l$. Put $k=\left[N^{1 / l}\right], s=[N / k]$, and $r=N-k s$. Also for a fixed $a=\left(a_{1}, \ldots, a_{l}\right) \in \mathbb{R}_{+}^{l}$ denote by $P_{1}(a)$ the rectangle $\left[-a_{1}, a_{1}\right] \times \ldots \times\left[-a_{l-1}, a_{l-1}\right]$. To obtain a required partition of $P(a)$ first we choose step by step points $-a_{l}=t_{0}<$ $t_{1}<\ldots<t_{k-1}<t_{k}=a_{l}$ such that

$$
\int_{\left[t_{i-1}, t_{i}\right] \times P_{1}(a)} d \eta(x)=\frac{s+1}{N} \int_{P(a)} d \eta(x)
$$

for all $i=1, \ldots, r$, and

$$
\int_{\left[t_{i-1}, t_{i}\right] \times P_{1}(a)} d \eta(x)=\frac{s}{N} \int_{P(a)} d \eta(x)
$$


for all $i=r+1, \ldots, k$. Clearly,

$$
\left|t_{i}-t_{i-1}\right| \leq \frac{2(s+1)}{N} M a_{l} \leq 4 N^{-1 / l} B M a_{1} .
$$

Consider the following measures on $P_{1}(a)$

$$
d \eta_{i}(x)=\alpha_{i}(x) d x
$$

where

$$
\alpha_{i}(x)=\alpha_{i}\left(x_{1}, \ldots, x_{l-1}\right)=\int_{t_{i-1}}^{t_{i}} \alpha\left(x_{1}, \ldots, x_{l}\right) d x_{l}
$$

for all $\left(x_{1}, \ldots, x_{l-1}\right) \in P_{1}(a)$ and $i=1, \ldots, k$. Clearly, each $\eta_{i}$ is $2 M^{2}-$ uniform. Hence, by induction assumption for each $i=1, \ldots, k$ and $N_{i} \in \mathbb{N}$ there exists a partition of $P_{1}$ into $N_{i}$ rectangles $P_{i, 1}, \ldots, P_{i, N_{i}}$ with facets parallel to the corresponding facets of $P_{1}(a)$ such that

$$
\int_{P_{i, j}} d \eta_{i}(x)=\frac{1}{N_{i}} \int_{P_{1}(a)} d \eta_{i}(x),
$$

and

$$
\operatorname{diam} P_{i, j} \leq C(l-1, B, M) a_{1} N_{j}^{-1 /(l-1)}
$$

for all $j=1, \ldots, N_{i}$. Choose $N_{i}=s+1$ for $i=1, \ldots, r$ and $N_{i}=s$ for $i=r+1, \ldots, k$. Consider the following partition of $P(a)$ into $N$ rectangles

$$
P_{i, j} \times\left[t_{i-1}, t_{i}\right], \quad i=1, \ldots, k, j=1, \ldots, N_{i} .
$$

By (8) and (9) we immediately get that

$$
\int_{P_{i, j} \times\left[t_{i-1}, t_{i}\right]} d \eta(x)=\int_{P_{i, j}} d \eta_{i}(x)=\frac{1}{N_{i}} \int_{P_{1}(a)} d \eta_{i}(x)=\frac{1}{N} \int_{P(a)} d \eta(x)
$$

for all $i=1, \ldots, k$ and $j=1, \ldots, N_{i}$. So, for this partition (5) holds. Finally, combining (7) with (10) we get (6) for some constant $C(l, B, M)$. Lemma 1 is proved. 
Now we are ready to construct the required convex area-regular partitions. Proof of Proposition 11: We may assume that $N>8 d^{2}$. First we consider the case when $N$ is even. For a $(d+1)$-rectangle $P(a), a \in S^{d}$, denote by $F_{2 i-1}(a)$ its facet $x_{i}=a_{i}$ and by $F_{2 i}(a)$ its facet $x_{i}=-a_{i}, i=1, \ldots, d+1$. One can naturally associate with $P(a)$ a convex partition $\left\{R_{1}(a), \ldots, R_{2 d+2}(a)\right\}$ of $S^{d}$, where $R_{i}(a)=g\left(F_{i}(a)\right)$, and $g(x)=x /|x|$ for all $x \in \mathbb{R}^{d+1} \backslash\{0\}$.

Consider a one-parametric family of $(d+1)$-rectangles $P\left(a_{\lambda}\right)$, where

$$
a_{\lambda}=\left(\lambda, \sqrt{\frac{1-\lambda^{2}}{d}}, \ldots, \sqrt{\frac{1-\lambda^{2}}{d}}\right)
$$

Now we will choose such a $\lambda=\lambda(N)$ that our required convex area-regular partition could be obtained as a subpartition of $\left\{R_{1}\left(a_{\lambda}\right), \ldots, R_{2 d+2}\left(a_{\lambda}\right)\right\}$. Consider the function $G(\lambda)=\mu_{d}\left(R_{1}\left(a_{\lambda}\right)\right)$. Clearly, $G(1 / \sqrt{d+1})=1 /(2 d+2)$ (in this case $P\left(a_{\lambda}\right)$ is a hypercube). On the other hand $R_{1}\left(a_{\lambda}\right)$ is contained in the spherical cap $A((1,0, \ldots, 0)$, arccos $\lambda)$. Therefore, we can estimate $G(\lambda)$ from above as

$$
G(\lambda) \leq \mu_{d}(A((1,0, \ldots, 0), \arccos \lambda)) .
$$

Below we will use the following inequalities: for all $d \in \mathbb{N}$ and $N>8 d^{2}$

$\mu_{d}\left(A((1,0, \ldots, 0), \arccos (1-1 / 10 d)) \leq \frac{N / 2-d([N /(2 d+2)]+1)}{N} \leq \frac{1}{2 d+2}\right.$

The left hand side inequality is very rough. We need this inequality with any constant strictly less than 1 and depending only on $d$ in place of $1-1 / 10 d$. Now, by continuity of $G$ there exists

$$
\lambda \in[1 / \sqrt{d+1}, 1-1 / 10 d]
$$

such that

$$
G(\lambda)=\frac{N / 2-d([N /(2 d+2)]+1)}{N} .
$$

By symmetry arguments $\mu_{d}\left(R_{1}\left(a_{\lambda}\right)\right)=\mu_{d}\left(R_{2}\left(a_{\lambda}\right)\right)$ and

$$
\mu_{d}\left(R_{3}\left(a_{\lambda}\right)\right)=\ldots=\mu_{d}\left(R_{2 d+2}\left(a_{\lambda}\right)\right)=\frac{[N /(2 d+2)]+1}{N} .
$$


For each $i=1, \ldots, 2 d+2$ consider the unique measure $\eta_{i}$ on $F_{i}\left(a_{\lambda}\right)$ such that $\eta_{i}(E)=\mu_{d}(g(E))$ for each measurable set $E \subset F_{i}\left(a_{\lambda}\right)$ (this is indeed a measure, since $g$ is one-to-one). Clearly, (111) implies that each $\eta_{i}$ is $M_{d^{-}}$ uniform for large enough $M_{d}$. Choose

$$
N_{i}=\frac{N / 2-d([N /(2 d+2)]+1)}{N} \quad \text { for } i=1,2
$$

and

$$
N_{i}=\frac{[N /(2 d+2)]+1}{N} \text { for } i=3, \ldots, 2 d+2 .
$$

Now applying Lemma 1 for each $d$-rectangle $F_{i}\left(a_{\lambda}\right)$ with measure $\eta_{i}, i=$ $1, \ldots, 2 d+2$ we can get corresponding partition of $F_{i}\left(a_{\lambda}\right)$ into $N_{i}$ rectangles $P_{i, j}$ such that

$$
\int_{P_{i, j}} d \eta_{i}(x)=\frac{1}{N_{i}} \int_{F_{i}\left(a_{\lambda}\right)} d \eta_{i}(x)
$$

for $j=1, \ldots, N_{i}$. Moreover,

$$
\operatorname{diam} P_{i, j} \leq C_{d} N^{-1 / d}
$$

By its definition $\mu_{d}\left(g\left(P_{i, j}\right)\right)=1 / N, i=1, \ldots, 2 d+2, j=1, \ldots, N_{i}$. Now we observe that each $g\left(P_{i, j}\right)$ is a geodesically convex closed set. Indeed, the image under the map $g$ of a line segment contained in $F_{i}$ is a geodesic arc on sphere $S^{d}$. Therefore, the image of the convex set $P_{i, j}$ is geodesically convex. Finally, the estimate $\operatorname{diam} g\left(P_{i, j}\right) \leq C_{d} N^{-1 / d}$ follows from (11) and (12).

Now it remains to prove the proposition in the case when $N$ is odd. To this end we apply the same argument, with only difference that we replace $(d+1)$-rectangles $P\left(a_{\lambda}\right)$ by another family of polytopes $Q_{\lambda, \mu}$. Namely, for $\lambda, \mu \in(0,1)$ let $Q_{\lambda, \mu}$ be the convex hull of $2^{d+1}$ vertices

$$
\left(\lambda, \pm \sqrt{\frac{1-\lambda^{2}}{d}}, \ldots, \pm \sqrt{\frac{1-\lambda^{2}}{d}}\right), \quad\left(-\mu, \pm \sqrt{\frac{1-\mu^{2}}{d}}, \ldots, \pm \sqrt{\frac{1-\mu^{2}}{d}}\right) .
$$

Consider the map $\phi:[-1,1]^{d+1} \rightarrow Q_{\lambda, \mu}$ given by

$$
\phi\left(t_{1}, t\right):=\left(t_{1} \cdot \frac{\lambda+\mu}{2}+\frac{\lambda-\mu}{2},\left(t_{1} \cdot \frac{\sqrt{1-\lambda^{2}}+\sqrt{1-\mu^{2}}}{2 \sqrt{d}}+\frac{\sqrt{1-\lambda^{2}}-\sqrt{1-\mu^{2}}}{2 \sqrt{d}}\right) t\right),
$$


where $t_{1} \in[-1,1]$ and $t \in[-1,1]^{d}$. Let $F_{1}, \ldots, F_{2 d+2}$ be the facets of $[-1,1]^{d+1}$ numbered as before. As in the case of even $N$ we can choose $\lambda \in[1 / \sqrt{d+1}, 1-1 / 10 d]$ such that

$$
\mu_{d}\left(g \circ \phi\left(F_{1}\right)\right)=\frac{(N-1) / 2-d([N /(2 d+2)]+1)}{N}
$$

and then choose $\mu \in[1 / \sqrt{d+1}, 1-1 / 10 d]$ such that

$$
\mu_{d}\left(g \circ \phi\left(F_{2}\right)\right)=\frac{(N+1) / 2-d([N /(2 d+2)]+1)}{N} .
$$

Now by the symmetry argument for $i=3, \ldots, 2 d+2$

$$
\mu_{d}\left(g \circ \phi\left(F_{i}\right)\right)=\frac{[N /(2 d+2)]+1}{N} .
$$

Consider the pull-back measures $\eta_{i}$ on $F_{i}$ defined by $\eta_{i}(E):=\mu_{d}(g \circ \phi(E))$ for any measurable subset $E \subset F_{i}$ (this is a well-defined measure, since $g \circ \phi$ is a.e. one-to-one). Clearly, each $\eta_{i}$ is $M_{d}$-uniform for large enough $M_{d}$, $i=1, \ldots, 2 d+2$. Applying again Lemma 1 to the measures $\eta_{i}$ we get the corresponding area-regular partition of $S^{d}$. Also, the map $\phi$ has a useful property that the image of a hyperplane parallel to a facet of $[-1,1]^{d+1}$ is again a hyperplane. Therefore the partition is convex. Finally Lemma 1 provides that the diameter of this partition is at most $K_{d} N^{-1 / d}$ for some $K_{d}$ large enough.

Remark. The fact that $\mathcal{R}$ is convex easily implies that each $R_{i}, i=1, \ldots, N$, contains a spherical cap of radius $b_{d} N^{-1 / d}$.

The following Theorem $\mathrm{C}$ states that an arbitrary large enough and well distributed set of points is "almost" an equal weight quadrature formula in $S^{d}$; see [14, Theorem 3.1].

Theorem C. There exist constants $r_{d}>0$ and $B_{d}>0$ such that for each integer $m>B_{d}$, each $\eta \in(0,1)$, an arbitrary convex area-regular partition $\mathcal{R}=\left\{R_{1}, \ldots, R_{N}\right\}$ with $\|\mathcal{R}\|<\eta \frac{r_{d}}{m}$, and each collection of points $x_{i} \in R_{i}$, $i=1, \ldots, N$, the following inequalities

$$
(1-\eta) \int_{S^{d}}|P(x)| d \mu_{d}(x) \leq \frac{1}{N} \sum_{i=1}^{N}\left|P\left(x_{i}\right)\right| \leq(1+\eta) \int_{S^{d}}|P(x)| d \mu_{d}(x),
$$


hold for all polynomials $P$ of total degree at most $m$.

To prove Theorem 1 we need the following lemma.

Lemma 2. For each $\eta \in(0,1)$, an arbitrary convex area-regular partition $\mathcal{R}=\left\{R_{1}, \ldots, R_{N}\right\}$ with $\|\mathcal{R}\|<\eta \frac{r_{d}}{m+1}$, and any two collections of points $x_{i}, y_{i} \in R_{i}, i=1, \ldots, N$, the following inequalities

$$
\frac{1}{N} \sum_{i=1}^{N}\left|\nabla P\left(x_{i}\right)-\nabla P\left(y_{i}\right)\right| \leq 8 d \eta \int_{S^{d}}|\nabla P(x)| d \mu_{d}(x),
$$

$(1-8 d \eta) \int_{S^{d}}|\nabla P(x)| d \mu_{d}(x)<\frac{1}{N} \sum_{i=1}^{N}\left|\nabla P\left(x_{i}\right)\right|<(1+8 d \eta) \int_{S^{d}}|\nabla P(x)| d \mu_{d}(x)$,

hold for all polynomials $P$ of total degree $m \geq B_{d}$. The constants $r_{d}$ and $B_{d}$ are given by Theorem $C$.

Proof. First we will prove (14). Since $|\nabla P|=\sqrt{P_{1}^{2}+\ldots+P_{d+1}^{2}}$, where $P_{i} \in \mathcal{P}_{m+1}$ for $i=1, \ldots, d+1$, we have

$$
\frac{1}{N} \sum_{i=1}^{N}\left|\nabla P\left(x_{i}\right)-\nabla P\left(y_{i}\right)\right| \leq \frac{1}{N} \sum_{i=1}^{N} \sum_{j=1}^{d+1}\left|P_{j}\left(x_{i}\right)-P_{j}\left(y_{i}\right)\right| .
$$

Now, for each $Q \in \mathcal{P}_{m+1}$ we will estimate the value

$$
\frac{1}{N} \sum_{i=1}^{N}\left|Q\left(x_{i}\right)-Q\left(y_{i}\right)\right|
$$

Let $I_{1}$ be the set of indexes $i=1, \ldots, N$ such that the value $Q(x)$ has the same sign for all $x \in R_{i}$, and $I_{2}$ be the set of all other indexes, that is the set of $i=1, \ldots, N$, for which there exists a point $x \in R_{i}$ with $Q(x)=0$. Let $I_{3}$ be the set of indexes $i=1, \ldots, N$ such that $\left|Q\left(x_{i}\right)\right| \geq\left|Q\left(y_{i}\right)\right|$. Put $z_{i}:=x_{i}$, if $i \in I_{3}$ and $z_{i}:=y_{i}$ otherwise. Put $t_{i}:=y_{i}$, if $i \in I_{1} \cap I_{3}$, and $t_{i}:=x_{i}$, if $i \in I_{1} \backslash I_{3}$. For $i \in I_{2}$, let $t_{i}$ be a point in $R_{i}$ such that $Q\left(t_{i}\right)=0$. We have

$$
\frac{1}{N} \sum_{i=1}^{N}\left|Q\left(x_{i}\right)-Q\left(y_{i}\right)\right|=\frac{1}{N} \sum_{i \in I_{1}}\left|Q\left(z_{i}\right)\right|-\frac{1}{N} \sum_{i \in I_{1}}\left|Q\left(t_{i}\right)\right|+\frac{1}{N} \sum_{i \in I_{2}}\left|Q\left(x_{i}\right)-Q\left(y_{i}\right)\right|
$$




$$
\begin{aligned}
\leq \frac{1}{N} \sum_{i \in I_{1}}\left|Q\left(z_{i}\right)\right| & -\frac{1}{N} \sum_{i \in I_{1}}\left|Q\left(t_{i}\right)\right|+\frac{2}{N} \sum_{i \in I_{2}}\left|Q\left(z_{i}\right)\right|-\frac{2}{N} \sum_{i \in I_{2}}\left|Q\left(t_{i}\right)\right| \\
& \leq \frac{2}{N} \sum_{i=1}^{N}\left|Q\left(z_{i}\right)\right|-\frac{2}{N} \sum_{i=1}^{N}\left|Q\left(t_{i}\right)\right|,
\end{aligned}
$$

where $z_{i}, t_{i} \in R_{i}, i=1, \ldots, N$. Thus, by (13) we have

$$
\frac{1}{N} \sum_{i=1}^{N}\left|Q\left(x_{i}\right)-Q\left(y_{i}\right)\right| \leq 4 \eta \int_{S^{d}}|Q(x)| d \mu_{d}(x)
$$

So, the inequality (16) implies

$\frac{1}{N} \sum_{i=1}^{N}\left|\nabla P\left(x_{i}\right)-\nabla P\left(y_{i}\right)\right| \leq 4 \eta \sum_{j=1}^{d+1} \int_{S^{d}}\left|\nabla P_{j}(x)\right| d \mu_{d}(x) \leq 8 d \eta \int_{S^{d}}|\nabla P(x)| d \mu_{d}(x)$.

This proves (14). Now by the mean value theorem there exist $y_{i} \in R_{i}$ such that

$$
\frac{1}{N}\left|\nabla P\left(y_{i}\right)\right|=\int_{R_{i}}|\nabla P(x)| d \mu_{d}(x), \quad i=1, \ldots, N .
$$

Finally, we obtain the inequality (15) from (14) and the following easy inequalities

$$
\begin{gathered}
\frac{1}{N} \sum_{i=1}^{N}\left|\nabla P\left(y_{i}\right)\right|-\frac{1}{N} \sum_{i=1}^{N}\left|\nabla P\left(x_{i}\right)-\nabla P\left(y_{i}\right)\right| \leq \frac{1}{N} \sum_{i=1}^{N}\left|\nabla P\left(x_{i}\right)\right| \\
\leq \frac{1}{N} \sum_{i=1}^{N}\left|\nabla P\left(y_{i}\right)\right|+\frac{1}{N} \sum_{i=1}^{N}\left|\nabla P\left(x_{i}\right)-\nabla P\left(y_{i}\right)\right| .
\end{gathered}
$$

The following lemma is crucial to construct the maps $\mathbf{x}_{1}, \ldots, \mathbf{x}_{N}: \mathcal{P}_{t} \rightarrow$ $S^{d}$ in the next section.

Lemma 3. For $x \in S^{d}$ denote by $T_{x}$ the space of all vectors $y \in \mathbb{R}^{d+1}$ with $(x, y)=0$. Let $R \subset S^{d}$ be a closed geodesically convex set with $\operatorname{diam} R<\pi / 2$. Then for each interior point $x \in R$ and $y \in T_{x} \backslash\{0\}$ the following holds: 
(i) there exists a unique $x_{\max } \in R$ with $\left(x_{\max }, y\right)=\max _{z \in R}(z, y)$;

(ii) the map $M_{x}: T_{x} \backslash\{0\} \rightarrow R$ given by $y \rightarrow x_{\max }$ is continuous on $T_{x} \backslash\{0\}$;

(iii) for each $w \in R$ and a geodesic $\gamma:[0,1] \rightarrow R$ with $\gamma(0)=x_{\max }, \gamma(1)=w$ the function $(y, \gamma(h))$ is decreasing on $[0,1]$.

Proof. Consider an orthogonal projection $p: \mathbb{R}^{d+1} \rightarrow T_{x}$ given by

$$
p(z)=z-(x, z) x .
$$

Clearly,

$$
(z, y)=(p(z), y)
$$

for all $z \in R$.

Denote by $S=p(R)$ the image of $R$ under the projection $p$. Since $\operatorname{dist}(x, z)<\pi / 2$ for each $z \in R$, then $p$ is a homeomorphism between $R$ and $S$ and the inverse map is given by

$$
p^{-1}(u)=u+\sqrt{1-|u|^{2}} x, \quad u \in S
$$

Now we will show that $S$ is a strictly convex subset of $T_{x}$, i.e. for each pair of distinct points $u, v \in S$ and each $h \in(0,1)$ the point $h u+(1-h) v$ is an interior point of $S$. To this end we note that

$$
p^{-1}(h u+(1-h) v)=h p^{-1}(u)+(1-h) p^{-1}(v)+\alpha x
$$

where $\alpha>0$. We will use the following simple statement:

If $w_{1}, w_{2} \in S^{d}$ are such that $\left(w_{1}, w_{2}\right)>0$, and $w_{3}=\alpha_{1} w_{1}+\alpha_{2} w_{2} \in S^{d}$ for some $\alpha_{1}, \alpha_{2}>0$, then $w_{3}$ lies on the shortest geodesic connecting $w_{1}$ and $w_{2}$. This statement and the fact that $p^{-1}(u), p^{-1}(v) \in R$ immediately imply that $z /|z| \in R$, where $z=h p^{-1}(u)+(1-h) p^{-1}(v)$. Hence, applying again the statement for $z /|z|$ and $x$ we get by (18) that $p^{-1}(h u+(1-h) v) \in R$. Moreover, since $x$ is an interior point of $R$, and $\alpha>0$, then $p^{-1}(h u+(1-h) v)$ is an interior point of $R$ as well, and therefore $h u+(1-h) v$ is an interior point of $S$. 
To prove (i) we will use the known fact that a nonconstant linear function given on a closed strictly convex subset in $\mathbb{R}^{d}$ attains its maximum in a unique point. Using this fact we get that there exists a unique $z_{\max } \in S$ such that $\left(z_{\max }, y\right)=\max _{z \in S}(z, y)$. Finally, by (17) we get that $x_{\max }=p^{-1}\left(z_{\max }\right)$.

Now we will prove (ii). Since $p$ is a homeomorphism it suffices to show that the composition map $p \circ M_{x}: T_{x} \backslash\{0\} \rightarrow S$ is continuous. Note that $\left(y, p \circ M_{x}(y)\right)=\max _{z \in S}(y, z)$. Since $S$ is a closed strictly convex set then for each $\varepsilon>0$ there exists $\delta=\delta(\varepsilon)>0$ such that for all $v \in T_{x}$ with $|v-y|<\delta$ the diameter of the set $\left\{z \in S \mid(v, z)>\left(v, p\left(M_{x}(y)\right)\right)\right\}$ is less than $\varepsilon$. Hence, $\left|p\left(M_{x}(y)\right)-p\left(M_{x}(v)\right)\right|<\varepsilon$. Thus, the map $p \circ M_{x}$ is continuous at $y$, and so is $M_{x}$. This proves (ii).

Finally, we prove part (iii) of the lemma. Let $G$ be the great circle containing $x_{\max }$ and $w$. There is a unique point $w_{\max } \in G$ such that $\left(y, w_{\max }\right)=$ $\max _{z \in G}(y, z)$. Now for each $z \in G$ we have

$$
(y, z)=\left(y, w_{\max }\right)\left(z, w_{\max }\right) .
$$

Hence the scalar product $(y, z)$ is increasing on both geodesic arcs connecting $-w_{\max }$ and $w_{\max }$. The geodesic $\gamma:[0,1] \rightarrow R$ is an arc of $G$. To prove (iii) it is enough to show that both $-w_{\max }$ and $w_{\max }$ are outside of the arc $\gamma$. The point $w_{\max }$ is outside of $\gamma$ by the definition of $x_{\max }$. Moreover, $\left(y, x_{\max }\right)>(y, x)=0$. Therefore, substituting $z=x_{\max }$ to (19) we see that $\left(x_{\max }, w_{\max }\right)>0$. Hence,

$$
\operatorname{dist}\left(x_{\max },-w_{\max }\right)>\pi / 2 .
$$

Finally, the fact that $\operatorname{diam} R<\pi / 2$ implies that $-w_{\max }$ is outside of $\gamma$ as well. Thus, the function $(y, \gamma(h))$ is decreasing on $[0,1]$. 


\section{Proof of Theorem 1}

Fix $t \in \mathbb{N}$. In Section 2 we explained that it is enough to construct an $N$-tuple of continuous maps $\mathbf{x}_{1}, \ldots, \mathbf{x}_{N}: \mathcal{P}_{t} \rightarrow S^{d}$ such that

$$
\frac{1}{N} \sum_{i=1}^{N} P\left(\mathbf{x}_{i}(P)\right)>0
$$

for all $P \in \partial \Omega$ and

$$
\operatorname{dist}\left(\mathbf{x}_{i}(P), \mathbf{x}_{j}(P)\right)>\lambda_{d} N^{-1 / d}, \quad 1 \leq i<j \leq N
$$

for all $P \in \Omega$, where $\Omega$ is given by (4).

Fix $\varepsilon, \delta, \eta>0$. Consider the function

$$
g_{\varepsilon}(t):= \begin{cases}t / \varepsilon & \text { if } t \leq \varepsilon, \\ 1 & \text { otherwise. }\end{cases}
$$

Let $N>t^{d}\left(\frac{2 K_{d}}{\eta r_{d}}\right)^{d}$ and $\mathcal{R}=\left\{R_{1}, \ldots, R_{N}\right\}$ be an area-regular partition provided by Proposition 11, For each $i=1, \ldots, N$ choose a point $x_{i} \in R_{i}$ such that $R_{i}$ contains a spherical cap of radius $b_{d} N^{-1 / d}$ with center at $x_{i}$. Recall that $\|R\| \leq K_{d} N^{-1 / d}$.

Let $P \in \mathcal{P}_{t}$. By Lemma 3 for each $i=1, \ldots, N$ there exists a unique $z_{i}=z_{i}(P) \in R_{i}$ satisfying

$$
\left(z_{i}, \nabla P\left(x_{i}\right)\right)=\max _{x \in R_{i}}\left(x, \nabla P\left(x_{i}\right)\right),
$$

provided that $\nabla P\left(x_{i}\right) \neq 0$. In the case $\nabla P\left(x_{i}\right)=0$ put $z_{i}=x_{i}$. Let $\gamma_{\left[x_{i}, z_{i}\right]}:[0,1] \rightarrow R_{i}$ be a geodesic connecting $x_{i}$ and $z_{i}$. We assume that the curve $\gamma_{\left[x_{i}, z_{i}\right]}$ has an equal-speed parametrization, i.e. the derivative with respect to parameter $h$ satisfies $\left|\gamma_{\left[x_{i}, z_{i}\right]}^{\prime}(h)\right|=\operatorname{dist}\left(x_{i}, z_{i}\right)$ for $h \in(0,1)$. Define

$$
\mathbf{x}_{i}(P):=\gamma_{\left[x_{i}, z_{i}\right]}\left((1-\delta) g_{\varepsilon}\left(\left|\nabla P\left(x_{i}\right)\right|\right)\right)
$$

By the definition of $g_{\varepsilon}$ the map $\mathbf{x}_{i}: \mathcal{P}_{t} \rightarrow S^{d}$ is continuous in a small neighborhood of the set $\left\{P \in \mathcal{P}_{t} \mid \nabla P\left(x_{i}\right)=0\right\}$. On the other hand, part (ii) 
of Lemma 3 implies that $\mathbf{x}_{i}$ is continuous on the set $\left\{P \in \mathcal{P}_{t}: \nabla P\left(x_{i}\right) \neq 0\right\}$. Thus the maps $\mathbf{x}_{1}, \ldots \mathbf{x}_{N}$ are continuous in $\mathcal{P}_{t}$. The following Lemma 4 will finish the proof of Theorem 1 .

Lemma 4. There exist constants $\varepsilon, \delta, \eta$ depending only on $d$ such that for each $N>t^{d}\left(\frac{2 K_{d}}{\eta r_{d}}\right)^{d}$ the $N$-tuple of maps $\mathbf{x}_{1}, \ldots, \mathbf{x}_{N}: \mathcal{P}_{t} \rightarrow S^{d}$ defined by (21) satisfies the following properties:

$$
\frac{1}{N} \sum_{i=1}^{N} P\left(\mathbf{x}_{i}(P)\right)>0
$$

for all $P \in \partial \Omega$ and

$$
\operatorname{dist}\left(\mathbf{x}_{i}(P), \mathbf{x}_{j}(P)\right)>\lambda_{d} N^{-1 / d}, \quad 1 \leq i<j \leq N,
$$

for all $P \in \Omega$.

Proof. Fix $P \in \mathcal{P}_{t}$. For each $i=1, \ldots, N$ choose $z_{i, \max } \in R_{i}$ such that $P\left(z_{i, \max }\right)=\max _{x \in R_{i}} P(x)$. Denote $y_{i, \varepsilon}:=\gamma_{\left[x_{i}, z_{i}\right]}\left(g_{\varepsilon}\left(\left|\nabla P\left(x_{i}\right)\right|\right)\right)$, where $z_{i}$ and $\gamma$ are as in (21). We can split the sum (22) into four pieces

$$
\begin{aligned}
\frac{1}{N} \sum_{i=1}^{N} P\left(\mathbf{x}_{i}(P)\right) & =\frac{1}{N} \sum_{i=1}^{N} P\left(z_{i, \max }\right) \\
& +\frac{1}{N} \sum_{i=1}^{N}\left(P\left(z_{i}\right)-P\left(z_{i, \max }\right)\right) \\
& +\frac{1}{N} \sum_{i=1}^{N}\left(P\left(y_{i, \varepsilon}\right)-P\left(z_{i}\right)\right) \\
& +\frac{1}{N} \sum_{i=1}^{N}\left(P\left(\mathbf{x}_{i}(P)\right)-P\left(y_{i, \varepsilon}\right)\right) .
\end{aligned}
$$

We will estimate each of these sums separately.

Clearly,

$$
\frac{1}{N} \sum_{i=1}^{N} P\left(z_{i, \max }\right)=\sum_{i=1}^{N} \int_{R_{i}}\left(P\left(z_{i, \max }\right)-P(x)\right) d \mu_{d}(x) .
$$


Now note that if $z_{i, \max } \notin \partial R_{i}$ for some $i=1, \ldots, N$ then $\nabla P\left(z_{i, \max }\right)=0$, therefore

$$
P\left(z_{i, \max }\right)-P(x) \geq \min _{y \in R_{i}}|\nabla P(y)| \operatorname{dist}\left(x, \partial R_{i}\right)
$$

for all $i=1, \ldots, N$ and $x \in R_{i}$. Let $A_{i}$ be a spherical cap of radius $b_{d} N^{-1 / d}$ contained in $R_{i}$. Since $\|R\| \leq K_{d} N^{-1 / d}$ we obtain that

$$
\int_{R_{i}} \operatorname{dist}\left(x, \partial R_{i}\right) d \mu_{d}(x) \geq \int_{A_{i}} \operatorname{dist}\left(x, \partial A_{i}\right) d \mu_{d}(x) \geq \beta_{d} \frac{\|\mathcal{R}\|}{N},
$$

for some constant $\beta_{d}$. Thus, it follows from (25) and (26) that

$$
\frac{1}{N} \sum_{i=1}^{N} P\left(z_{i, \max }\right) \geq \beta_{d} \frac{\|\mathcal{R}\|}{N} \sum_{i=1}^{N} \min _{y \in R_{i}}|\nabla P(y)| .
$$

Since by Theorem 1 we have $\|\mathcal{R}\|<\eta r_{d} /(t+1)$, using Lemma 2 we arrive at

$$
\frac{1}{N} \sum_{i=1}^{N} P\left(z_{i, \max }\right) \geq\|\mathcal{R}\| \beta_{d}(1-8 d \eta) \int_{S^{d}}|\nabla P(x)| d \mu_{d}(x) .
$$

Next we estimate the sum

$$
\frac{1}{N} \sum_{i=1}^{N}\left(P\left(z_{i, \max }\right)-P\left(z_{i}\right)\right)
$$

Let $\gamma:[0,1] \rightarrow R_{i}$ be a geodesic connecting $z_{i}$ and $z_{i, \max }$. We can write

$$
P\left(z_{i, \max }\right)-P\left(z_{i}\right)=\int_{0}^{1}\left(\nabla P(\gamma(h)), \gamma^{\prime}(h)\right) d h \geq 0 .
$$

By Lemma 3 (iii) the inequality $\left(\gamma^{\prime}(h), \nabla P\left(x_{i}\right)\right)<0$ holds for all $h \in(0,1)$. Thus, we have

$$
\begin{gathered}
\int_{0}^{1}\left(\nabla P(\gamma(h)), \gamma^{\prime}(h)\right) d h \leq \int_{0}^{1}\left(\nabla P(\gamma(h))-\nabla P\left(x_{i}\right), \gamma^{\prime}(h)\right) d h \\
\leq \int_{0}^{1}\left|\nabla P(\gamma(h))-\nabla P\left(x_{i}\right)\right|\left|\gamma^{\prime}(h)\right| d h \leq \operatorname{diam} R_{i} \max _{x \in R_{i}}\left|\nabla P(x)-\nabla P\left(x_{i}\right)\right| .
\end{gathered}
$$


Using Lemma 2 we arrive at

$$
\frac{1}{N} \sum_{i=1}^{N}\left|P\left(z_{i, \max }\right)-P\left(z_{i}\right)\right| \leq\|\mathcal{R}\| 8 d \eta \int_{S^{d}}|\nabla P(x)| d \mu_{d}(x) .
$$

Now we estimate the third sum in the left-hand side of (24). Recall that

$$
y_{i, \varepsilon}= \begin{cases}z_{i} & \text { for }\left|\nabla P\left(x_{i}\right)\right| \geq \varepsilon \\ \gamma_{\left[x_{i}, z_{i}\right]}\left(\left|\nabla P\left(x_{i}\right)\right| / \varepsilon\right) & \text { otherwise. }\end{cases}
$$

Hence, we obtain

$$
\frac{1}{N} \sum_{i=1}^{N}\left(P\left(y_{i, \varepsilon}\right)-P\left(z_{i}\right)\right)=\frac{1}{N} \sum_{i:\left|\nabla P\left(x_{i}\right)\right|<\varepsilon}\left(P\left(y_{i, \varepsilon}\right)-P\left(z_{i}\right)\right) .
$$

Since $z_{i}$ and $y_{i, \varepsilon}$ are both in $R_{i}$, we can write an obvious estimate

$$
\left|P\left(y_{i, \varepsilon}\right)-P\left(z_{i}\right)\right| \leq \operatorname{diam} R_{i} \max _{x \in R_{i}}|\nabla P(x)| .
$$

For each $i=1, \ldots, N$ choose $w_{i} \in R_{i}$ such that $\left|\nabla P\left(w_{i}\right)\right|=\max _{x \in R_{i}}|\nabla P(x)|$. Then

$$
\begin{gathered}
\frac{1}{N} \sum_{i:\left|\nabla P\left(x_{i}\right)\right|<\varepsilon}\left|\nabla P\left(w_{i}\right)\right| \leq \\
\frac{1}{N} \sum_{i:\left|\nabla P\left(x_{i}\right)\right|<\varepsilon}\left(\left|\nabla P\left(w_{i}\right)\right|-\left|\nabla P\left(x_{i}\right)\right|\right)+\varepsilon+\frac{1}{N} \sum_{i:\left|\nabla P\left(x_{i}\right)\right| \geq \varepsilon}\left(\left|\nabla P\left(x_{i}\right)\right|-\left|\nabla P\left(x_{i}\right)\right|\right) .
\end{gathered}
$$

Thus, Lemma 2 implies that

$$
\frac{1}{N} \sum_{i:\left|\nabla P\left(x_{i}\right)\right|<\varepsilon}\left|\nabla P\left(w_{i}\right)\right| \leq \varepsilon+8 d \eta \int_{S^{d}}|\nabla P(x)| d \mu_{d}(x) .
$$

Hence, we arrive at

$$
\left|\frac{1}{N} \sum_{i=1}^{N}\left(P\left(y_{i, \varepsilon}\right)-P\left(z_{i}\right)\right)\right| \leq\|\mathcal{R}\|\left(\varepsilon+8 d \eta \int_{S^{d}}|\nabla P(x)| d \mu_{d}(x)\right) .
$$


It remains to estimate the sum

$$
\frac{1}{N} \sum_{i=1}^{N}\left(P\left(\mathbf{x}_{i}(P)\right)-P\left(y_{i, \varepsilon}\right)\right) .
$$

The distance between $\mathbf{x}_{i}(P)$ and $y_{i, \varepsilon}$ is less than $\delta\|\mathcal{R}\|$. Hence,

$$
\left|\frac{1}{N} \sum_{i=1}^{N}\left(P\left(\mathbf{x}_{i}(P)\right)-P\left(y_{i, \varepsilon}\right)\right)\right| \leq \frac{\delta\|\mathcal{R}\|}{N} \sum_{i=1}^{N} \max _{x \in R_{i}}|\nabla P(x)| .
$$

Using again Lemma 2 we arrive at

$$
\left|\frac{1}{N} \sum_{i=1}^{N}\left(P\left(\mathbf{x}_{i}\right)-P\left(y_{i, \varepsilon}\right)\right)\right| \leq\|\mathcal{R}\| \delta(1+8 d \eta) \int_{S^{d}}|\nabla P(x)| d \mu_{d}(x) .
$$

Now for $P \in \partial \Omega$, we get by (24), (28), (29), (30), and (31) that

$$
\frac{1}{N} \sum_{i=1}^{N} P\left(\mathbf{x}_{i}(P)\right) \geq\|\mathcal{R}\|\left(\beta_{d}(1-8 d \eta)-8 d \eta-(8 d \eta+\varepsilon)-\delta(1+8 d \eta)\right)
$$

Take $\eta=\beta_{d} /(48 d), \delta=\beta_{d} / 3$ and $\varepsilon=\beta_{d} / 12$, where $\beta_{d}$ is provided by (27). Without loss of generality we may assume that $\beta_{d}<1$. Thus we get

$$
\beta_{d}(1-8 d \eta)-8 d \eta-(8 d \eta+\varepsilon)-\delta(1+8 d \eta)>0
$$

which together with (32) imply (22).

It remains to show the separation property (23). Fix $P \in \Omega$ and $i \in$ $\overline{1, \ldots, N}$. By the definition (21), $\mathbf{x}_{i}(P)$ is in $R_{i}$. Thus to prove (23) it is enough to show that $\operatorname{dist}\left(\mathbf{x}_{i}(P), \partial R_{i}\right) \geq \lambda_{d} N^{-1 / d}$ for some constant $\lambda_{d}$. Recall that $\mathbf{x}_{i}(0)=x_{i}$, and $R_{i}$ contains a spherical cap of radius $r=b_{d} N^{-1 / d}$ with center at $x_{i}$. The main reason why $x_{i}(P)$ is "far away" from the boundary $\partial R_{i}$ is because $\mathbf{x}_{i}(P)$ lies on the geodesic $\gamma_{\left[x_{i}, z_{i}\right]}$, where $z_{i} \in R_{i}$, and

$$
\operatorname{dist}\left(x_{i}, \mathbf{x}_{i}(P)\right) \leq(1-\delta) \operatorname{dist}\left(x_{i}, z_{i}\right)
$$

We will also use the fact that $R_{i}$ is geodesically convex and contains a spherical cap of "big" radius with center at $x_{i}$. 
Denote by $T_{x_{i}}$ the space of all vectors in $\mathbb{R}^{d+1}$ orthogonal to $x_{i}$ and let $p: \mathbb{R}^{d+1} \rightarrow T_{x_{i}}$ be the orthogonal projection

$$
p(z)=z-\left(x_{i}, z\right) x_{i} .
$$

As we have pointed out in Lemma 3 the image $S_{i}=p\left(R_{i}\right)$ is a convex subset in $T_{x_{i}}$. Clearly,

$$
\operatorname{dist}\left(\mathbf{x}_{i}(P), \partial R_{i}\right) \geq \operatorname{dist}_{\text {euc }}\left(p\left(\mathbf{x}_{i}(P)\right), \partial S_{i}\right),
$$

where $\operatorname{dist}_{\text {euc }}\left(z, \partial S_{i}\right)$ stands for the Euclidean distance between point $z$ and the set $\partial S_{i}$ in $T_{x_{i}}$. The point $p\left(\mathbf{x}_{i}(P)\right)$ lies between the points $p\left(x_{i}\right)=0$ and $p\left(z_{i}\right)$ on the line connecting them. Thus we have

$$
\left|p\left(x_{i}\right)-p\left(z_{i}\right)\right|=\sin \left(\operatorname{dist}\left(x_{i}, z_{i}\right)\right),
$$

and

$$
\left|p\left(x_{i}\right)-p\left(\mathbf{x}_{i}(P)\right)\right| \leq \sin \left((1-\delta) \operatorname{dist}\left(x_{i}, z_{i}\right)\right) .
$$

Moreover, the fact that $R_{i}$ contains the spherical cap $A\left(x_{i}, r\right)$ implies that

$$
\operatorname{dist}_{\text {euc }}\left(p\left(x_{i}\right), \partial S_{i}\right) \geq \sin r .
$$

Finally we note that the function $\operatorname{dist}_{\mathrm{euc}}\left(z, \partial S_{i}\right)$ is concave on $S_{i}$. Therefore by (33)-(35) we get

$$
\operatorname{dist}\left(\mathbf{x}_{i}(P), \partial R_{i}\right) \geq \frac{\delta}{2} \sin r \geq \lambda_{d} N^{-1 / d},
$$

which implies (23). Lemma 4 is proved.

Acknowledgements. The authors thank the Mathematisches Forschungsinstitut Oberwolfach for their hospitality during the preparation of this manuscript and for providing a stimulating atmosphere for research. This paper is partially supported by the Centre for Advanced Study at the Norwegian Academy of Science and Letters in Oslo. 


\section{References}

[1] R. Alexander, On the sum of distances between $n$ points on a sphere, Acta Mathematica Hungarica 23 (1972), pp. 443-448.

[2] C. An, X. Chen, I. H. Sloan, And R. S. Womersley, Well conditioned spherical designs for integration and interpolation on the twosphere, SIAM Journal on Numerical Analysis 48 (2010), pp. 2135-2157.

[3] B. Bajnok, Construction of spherical t-designs, Geom. Dedicata, 43 (1992), pp. 167-179.

[4] A. Bondarenko, D. Radchenko, and M. Viazovska, Optimal asymptotic bounds for spherical designs, Annals of Mathematics, 178 (2013), pp. 443-452.

[5] A. Bondarenko, M. Viazovska, Spherical designs via Brouwer fixed point theorem, SIAM J. Discrete Math., 24 (2010), pp. 207-217.

[6] J. Bourgain, J. Lindenstrauss, Distribution of points on spheres and approximation by zonotopes, Israel J. Math., 64 (1988), pp. 25-31.

[7] X. Chen, R. S. Womersley, Existence of solutions to systems of underdetermined equations and spherical designs, SIAM Journal on Numerical Analysis, 44 (2006), pp. 2326 - 2341.

[8] J. H. Conway, N. J. A. Sloane, Sphere packings, lattices and groups., 3rd ed., Springer, New York, 1999.

[9] P. Delsarte, J. M. Goethals, and J. J. Seidel, Spherical codes and designs, Geom. Dedicata, 6 (1977), pp. 363-388.

[10] R. H. Hardin, N. J. A. Sloane, McLaren's Improved Snub Cube and Other New Spherical Designs in Three Dimensions, Discrete Comput. Geom., 15 (1996), pp. 429-441. 
[11] K. Hesse, P. Leopardi, The Coulomb energy of spherical designs, Adv. Comp. Math., 28 (2008), pp. 331-354.

[12] J. Korevane, J. L. H. Meyers, Spherical Faraday cage for the case of equal point charges and Chebyshev-type quadrature on the sphere, Integral Transforms Spec. Funct. 1 (1993), pp. 105-117.

[13] A. B. J. KuiJlaArs, E. B. SAFF, Asymptotics for minimal discrete energy on the sphere, Trans. Amer. Math. Soc., 350 (1998), pp. 523-538.

[14] H. N. Mhaskar, F. J. Narcowich, and J. D. Ward, Spherical Marcinkiewicz-Zygmund inequalities and positive quadrature, Math. Comp., 70 (2001), pp. 1113-1130.

[15] Donal O'Regan, Yeol Je Cho, and Yu Qing Chen, Topological degree theory and applications, Chapman \& Hall/CRC, 2006.

[16] E. B. SAfF, A. B. J. KuIJlaARs, Distributing many points on a sphere, Math. Intelligencer, 19 (1997), pp. 5-11.

[17] P. D. Seymour and T. Zaslavsky, Averaging sets, Adv. Math., 52 (1984), pp. 213-240.

[18] S. Smale, Mathematics: frontiers and perspectives, Amer. Math, Soc., Providence, (2000), pp. 271-294.

[19] G. Wagner, On averaging sets, Monatsh. Math., 111 (1991), pp. 69-78.

Department of Mathematical Analysis, Taras Shevchenko National University of Kyiv, Volodymyrska 64, 01033 Kyiv, Ukraine and

Department of Mathematical Sciences, Norwegian University of Science and Technology, NO-7491 Trondheim, Norway

Email address: andriybond@gmail.com

Max Planck Institute for Mathematics, Vivatsgasse 7, 53111 Bonn, Germany and 
Department of Mathematical Analysis, Taras Shevchenko National University of Kyiv, Volodymyrska 64, 01033 Kyiv, Ukraine

Email address: danrad@mpim-bonn.mpg.de

Max Planck Institute for Mathematics, Vivatsgasse 7, 53111 Bonn, Germany

Email address: viazovska@gmail.com 